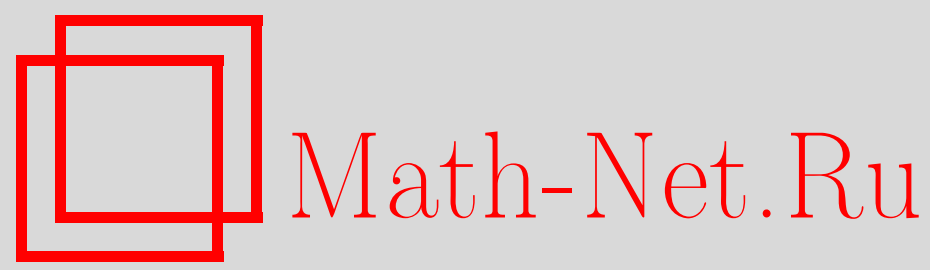

Н. В. Величко, О секвенциальной сепарабельности, Матем. заметки, 2005, том 78, выпуск 5, 652-657

DOI: https://doi.org/10.4213/mzm2639

Использование Общероссийского математического портала Math-Net.Ru подразумевает, что вы прочитали и согласны с пользовательским соглашением http://www .mathnet.ru/rus/agreement

Параметры загрузки:

IP : 54.80 .97 .219

26 апреля 2023 г., 17:36:54

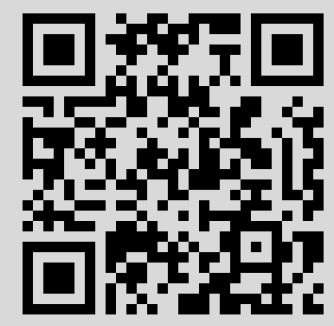




\section{О СЕКВЕНЦИАЛЬНОЙ СЕПАРАБЕЛЬНОСТИ}

\section{Н. В. Величко}

Получен новый критерий секвенциальной сепарабельности пространства $C(X)$ непрерывных вещественных функций на $X$ в топологии простой сходимости.

Библиограффия: 4 названия.

Секвенииально сепарабельным назьвается пространство, имеющее счетное секвенциально плотное в нем подмножество.

Множество $A$ секвенциально плотно в пространстве $X$, если каждая точка $x \in X$ является пределом некоторой последовательности точек $A$. Ясно, что каждое секвенциально сепарабельное пространство сепарабельно; обратное неверно.

Все пространства предполагаются тихоновскими.

Через $C_{p}(X)$ обозначается линейное пространство всех непрерьвных вещественных функций на пространстве $X$, наделенное топологией поточечной сходимости.

Через $\mathbb{R}$ и $\mathbb{N}$ обозначаются числовая прямая и множество всех положительных целых чисел.

Нуль-множество в $X$ - множество нулей некоторой непрерьвной вещественной функции на $X$, конуль-множество - дополнение до нуль-множества.

Известная проблема - когда пространство $C_{p}(X)$ секвенциально сепарабельно, недавно решена в работе [1].

В заметке предлагается другой критерий секвенциальной сепарабельности $C_{p}(X)$, основанньй на некоторых идеях и конструкциях теории $B$-измеримых отображений $[2$, $30,31]$.

В качестве приложения доказана секвенциальная сепарабельность $C_{p}(X)$ для двух конкретных пространств.

ТЕОрема 1. Пространство $C_{p}(X)$ секвенииально сепарабельно тогда и только тогда, когда существует уплотнение пространства $X$ на сеперабельное метризуемое пространство $Y$, являющееся бәровским изоморфизмом первого уровня (т.е. образ любого конуль-мнохсества из $X$ является $F_{\sigma}$-множеством в $Y$ ).

ДокАЗАТЕЛьСтво. Предположим, что $C_{p}(X)$ секвенциально сепарабельно. Пусть $S$ - счетное секвенциально плотное в $C_{p}(X)$ множество.

Наделяем $X$ слабой топологией $\tau$ относительно семейства $S$.

Напомним определение слабой топологии.

Пусть $\tau_{e}$ - естественная топология числовой прямой $\mathbb{R}$. Тогда $\tau$ есть точная верхняя грань семейства $\left\{f^{-1}\left(\tau_{e}\right)=\left\{f^{-1}(V): V \in \tau_{e}\right\}: f \in S\right\}$ прообразов топологии $\tau_{e}$ при 
отображениях из $S$. Базу топологии $\tau$ образуют всевозможные конечные пересечения элементов семейства $\left\{f^{-1}\left(\tau_{e}^{\prime}\right): f \in S, \tau_{e}^{\prime}\right.$ - счетная база $\left.\tau_{e}\right\}$, так что $\tau$ обладает счетной базой. Кроме того, пространство $Y=(X, \tau)$ регулярно, так что оно метризуемо по теореме Урысона.

Докажем, что требуемым уплотнением $F: X \rightarrow Y$ будет тождественное отображение $X$.

Так как всякая функция $f \circ F^{-1}$ непрерывна на $Y$ при $f \in S$, а при $f \in C(X)$ функция $f$ является поточечным пределом последовательности функций из $S$, то $f \circ F^{-1}$ является функцией первого класса на $Y$ [2,31.VIII.1], так что прообраз открытого в $\mathbb{R}$ множества при отображении $f \circ F^{-1}$ будет $F_{\sigma}$-множеством в $Y$.

Так как конуль-множество в $X$ является прообразом открытого в $\mathbb{R}$ множества, его образ при отображении $F$ будет $F_{\sigma}$-множеством в $Y$. Необходимость установлена.

Докажем достаточность. Пусть $F: X \rightarrow Z$ - уплотнение $X$ на сепарабельное метризуемое пространство $Z$, удовлетворяющее условиям теоремы. Пусть $f \in C(X)$. Можно предположить, что функция $f$ ограничена (в противном случае ее можно заменить функцией $\varphi \circ f$, где $\varphi: \mathbb{R} \rightarrow(0,1)$ - гомеоморфизм). Будем отождествлять любую функцию $g$ на $X$ с функцией $g \circ F^{-1}$ на $Z$ (отождествляя по существу множества (но не пространства) $X$ и $Z$ ).

Пусть $Y$-некоторое компактноеметризуемое расширениепространства $Z$. Для каждого $n \in \mathbb{N}$ по индукции построим

1) конечно-значную функцию $f_{n}$ на множестве $Z^{\prime} \supseteq Z$ такую, что выполняется соотношение

(a) $\left|f(x)-f_{n}(x)\right|<\frac{1}{2^{n+1}}$ при $x \in Z$;

2 ) последовательность $\left\{f_{n, m}: m \in \mathbb{N}\right\}$ непрерьвных функций на $Y$, поточечно сходящуюся на $Z$ к функции $f_{n}$ и такую, что вьполняется формула

(b) $\left|f_{n+1, m}(x)-f_{n, m}(x)\right| \leqslant \frac{1}{2^{n}}$.

Предположим, что такие объекты построены для всех $i<n$.

Выберем конечное покрытие $\left\{H_{n}^{i}: i \leqslant k(n)\right\}$ множества $f(X)$ интервалами длины $1 / 2^{n+1}$. Положим $V_{n}^{i}=f^{-1}\left(H_{n}^{i}\right)$. Так как $V_{n}^{i}$ - конуль-множество в пространстве $X$, то $F\left(V_{n}^{i}\right)$ будет $F_{\sigma}$-множеством в $Z$ и найдется $F_{\sigma}$-множество $W_{n}^{i}$ в $Y$ такое, что $W_{n}^{i} \cap Z=F\left(V_{n}^{i}\right)$ (если $F\left(V_{n}^{i}\right)=\bigcup\left\{F_{n, k}^{i}: k \in \mathbb{N}\right\}$, где $F_{n, k}^{i}$ замкнуты в $Z$, то $W_{n}^{i}$ можно определить как $\left.\cup\left\{\left[F_{n, k}^{i}\right]_{Y}: k \in \mathbb{N}\right\}\right)$. По теореме редукции [2,30.VIII.1] найдется дизъюнктное семейство $\left\{U_{n}^{i}\right\} \quad F_{\sigma}$-множеств в $Y$ такое, что $U_{n}^{i} \subseteq W_{n}^{i}$ и $\cup\left\{U_{n}^{i}: i \leqslant k(n)\right\}=$ $\cup\left\{W_{n}^{i}: i \leqslant k(n)\right\}$. Каждое $U_{n}^{i}$ можно представить как $\bigcup\left\{U_{n}^{i, m}: m \in \mathbb{N}\right\}$, где $U_{n}^{i, m}$ замкнуто в $Y$ и $U_{n}^{i, m} \subseteq U_{n}^{i, m+1}$. Для каждого $i$ выберем точку $x_{i} \in F\left(V_{n}^{i}\right)$ (произвольно) и положим $t_{i}=f\left(x_{i}\right)$. Тогда $t_{i} \in H_{n}^{i}$. Далее, полагая $f_{n}(x)=t_{i}$ при $x \in U_{n}^{i}$, получим конечно-значную функцию $f_{n}$ на множестве $\bigcup\left\{U_{n}^{i}: i \leqslant k(n)\right\} \supseteq Z$. Пусть $x \in Z$ - произвольная точка. Найдется $i$ такое, что $x \in U_{n}^{i}$. Так как $U_{n}^{i} \cap Z \subseteq F\left(V_{n}^{i}\right)$, то точки $f_{n}(x)$ и $f\left(x_{i}\right)$ находятся в одном интервале длины $1 / 2^{n+1}$; следовательно, $\left|f_{n}(x)-f(x)\right|<$ $1 / 2^{n+1}$. Это верно для каждого $x$, так что вьполняется (a).

Для каждого $m$ зададим функцию $g_{n, m}^{\prime}$ на множестве $A_{m}=\cup\left\{U_{n}^{i, m}: i \leqslant k(n)\right\}$, положив $g_{n, m}^{\prime}(x)=t_{i}$ при $x \in U_{n}^{i, m}$. Эта функция будет непрерьвной на $A_{m}$ в силу дизъюнктности семейства $\left\{U_{n}^{i, m}: i \leqslant k(n)\right\}$. Используя замкнутость $A_{m}$, применим теорему Урысона и продолжим $g_{n, m}^{\prime}$ по непрерывности до функции $g_{n, m}: Y \rightarrow \mathbb{R}$. Последовательность $\left\{g_{n, m}: m \in \mathbb{N}\right\}$ будет поточечно сходиться к функции $f_{n}$ на множес- 
тве $Z$. Докажем это.

Пусть $x \in Z$. Найдутся $i$ и $m$ такие, что $x \in U_{n}^{i, m}$. Тогда $g_{n, l}(x)=t_{i}$ для $l \geqslant m$, т.е. $g_{n, l}(x)=f_{n}(x)$. Рассмотрим непрерьвную функцию $g_{n, m}-f_{n-1, m}$. Заметим, принимая во внимание (a), что вьполняется

$$
\text { (c) }\left|f_{n}(x)-f_{n-1}(x)\right| \leqslant\left|f_{n}(x)-f(x)\right|+\left|f(x)-f_{n-1}(x)\right|<\frac{1}{2^{n+1}}+\frac{1}{2^{n}}<\frac{1}{2^{n-1}} \text {. }
$$

Положим

$$
h_{n, m}(x)= \begin{cases}\left(g_{n, m}-f_{n-1, m}\right)(x), & \text { если }\left(g_{n, m}-f_{n-1, m}\right)(x) \leqslant 1 / 2^{n-1}, \\ 1 / 2^{n-1}, & \text { если }\left(g_{n, m}-f_{n-1, m}\right)(x)>1 / 2^{n-1}, \\ -1 / 2^{n-1}, & \text { если }\left(g_{n, m}-f_{n-1, m}\right)(x)<-1 / 2^{n-1} .\end{cases}
$$

Положим $f_{n, m}=f_{n-1, m}+h_{n, m}$. Тогда последовательность непрерывных функций $\left\{f_{n, m}\right\}$ будет поточечно сходиться на множестве $Z$ к функции $f_{n}$. Действительно, пусть $x \in Z$. По (c) $\left|f_{n}(x)-f_{n-1}(x)\right|=\alpha<1 / 2^{n-1}$. Так как последовательность $\left\{g_{n, m}(x)\right\}$ сходится к $f_{n}(x)$, то найдется $k \in \mathbb{N}$ такое, что $\left|g_{n, l}(x)-f_{n}(x)\right|<\left(1 / 2^{n-1}-\alpha\right) / 2$ для $l \geqslant k$. Так как $\left\{f_{n-1, m}(x)\right\}$ сходится к $f_{n-1}(x)$, найдется $k_{1} \geqslant k$ такое, что $\mid f_{n-1, l}(x)-$ $f_{n-1}(x) \mid<\left(1 / 2^{n-1}-\alpha\right) / 2$ при $l \geqslant k_{1}$. Тогда при $l \geqslant k_{1}$ вьполняется

$$
\begin{aligned}
\left|g_{n, l}(x)-f_{n-1, l}(x)\right| & \leqslant\left|g_{n, l}(x)-f_{n}(x)\right|+\left|f_{n}(x)-f_{n-1}(x)\right|+\left|f_{n-1, l}(x)-f_{n-1}(x)\right| \\
& <\frac{1}{2}\left(\frac{1}{2^{n-1}}-\alpha\right)+\alpha+\frac{1}{2}\left(\frac{1}{2^{n-1}}-\alpha\right)=\frac{1}{2^{n-1}}-\alpha+\alpha=\frac{1}{2^{n-1}} .
\end{aligned}
$$

Из этого следует

$$
f_{n, l}(x)=f_{n-1, l}(x)+h_{n, l}(x)=f_{n-1, l}(x)+\left(g_{n, l}(x)-f_{n-1, l}\right)(x)=g_{n, l}(x) .
$$

Это означает, что последовательность $\left\{f_{n, m}(x)\right\}$ совпадает с последовательностью $\left\{g_{n, l}(x)\right\}$, начиная с некоторого номера, а так как последняя сходится к $f_{n}(x)$, то и $\left\{f_{n, m}(x)\right\}$ сходится к $f_{n}(x)$.

Также мы имеем

$$
\left|f_{n, m}(x)-f_{n-1, m}(x)\right|=\left|f_{n-1, m}(x)+h_{n, m}(x)-f_{n-1, m}(x)\right|=\left|h_{n, m}(x)\right| \leqslant \frac{1}{2^{n-1}},
$$

так что выполняется и (b).

Индукция проведена.

Положим $u_{n}=f_{n, n}$. Докажем, что $\left\{u_{n}\right\}$ поточечно сходится на $X$ к функции $f$. Доказательство этого факта будет проходить по стандарту теории $B$-измеримых отображений.

Пусть $x \in X-$ произвольная точка, $\varepsilon>0$. Выберем $n$ таким, чтобы $1 / 2^{n+1}<\varepsilon / 3$. В силу (а) $\left|f(x)-f_{n}(x)\right|<1 / 2^{n+1}$. Найдется $n_{0}>n$ такое, что $\left|f_{n}(x)-f_{n, m}(x)\right|<1 / 2^{n+1}$ при $m \geqslant n_{0}$ (в силу сходимости $\left\{f_{n, m}(x)\right\}$ к $\left.f_{n}(x)\right)$. Пусть $l \geqslant n_{0}$. Тогда, учитьвая (b), получаем следующую оценку:

$$
\begin{aligned}
\left|f(x)-u_{l}(x)\right| \leqslant & \left|f_{l, l}(x)-f_{l-1, l}(x)\right|+\cdots+\left|f_{n+1, l}(x)-f_{n, l}(x)\right| \\
& +\left|f_{n, l}(x)-f_{n}(x)\right|+\left|f_{n}(x)-f(x)\right| \\
< & \frac{1}{2^{l-1}}+\cdots+\frac{1}{2^{n}}+\frac{1}{2^{n+1}}+\frac{1}{2^{n+1}}<\frac{3}{2^{n+1}}<\varepsilon .
\end{aligned}
$$


Этим доказана сходимость $\left\{u_{n}(x)\right\}$ к $f(x)$ в точках $Z$.

Так как $Y$ - метризуемьй компакт, $C(Y)$ сепарабельно в топологии равномерной сходимости. Пусть $S$ - счетное плотное подмножество $C(Y)$. Для каждой функции $u_{n}$ выберем функцию $v_{n} \in S$ согласно условию $\left|u_{n}(x)-v_{n}(x)\right|<1 / n$ для всех $x \in Y$. Тогда последовательность $\left\{v_{n}\right\}$ будет поточечно сходиться к $f$ на множестве $Z$. Действительно, пусть $x \in Z$ и $\varepsilon>0$. Находим $n_{0}$ такое, что $\left|f(x)-u_{n}(x)\right|<\varepsilon / 2$ при $n>n_{0}$. Далее находим $n_{1} \geqslant n_{0}$ такое, что $1 / n<\varepsilon / 2$ при $n>n_{1}$. Тогда при $n>n_{1}$ вьполняется $\left|f(x)-v_{n}(x)\right| \leqslant\left|f(x)-u_{n}(x)\right|+\left|u_{n}(x)-v_{n}(x)\right|<\varepsilon / 2+\varepsilon / 2=\varepsilon$, что доказывает сходимость $\left\{v_{n}(x)\right\}$ к $f(x)$.

Теорема доказана.

Рассмотрим некоторые приложения теоремы 1.

СлЕДСТВИЕ 1. Если пространство $(X, T)$ нормально, то $C_{p}(X)$ секвенииально сепарабельно тогда и только тогда, когда существует уплотнение пространства $X$ на сеперабельное метризуемое пространство $Y$ такое, что образ любого открытого $F_{\sigma}$-множества из $(X, T)$ будет $F_{\sigma}$-множсеством в $\left(X, T^{\prime}\right)$.

Утверждение верно в силу того, что в нормальном пространстве конуль-множества совпадают с открытыми $F_{\sigma}$-множествами.

Обозначим через $B_{1}(X)$ пространство всех функций первого бэровского класса на сепарабельном метрическом пространстве $X$ в топологии поточечной сходимости. Тогда будет справедливым

СлЕдСТВИЕ 2. Пространство $B_{1}(X)$ секвенииально сепарабельно.

Следствие доказывается практически так же, как достаточность в теореме 1.

Очень просто доказывается

ТЕорема 2. Если пространство X псевдокомпактно, то следующие утверждения әквивалентны:

(a) пространство $C_{p}(X)$ сепарабельно;

(b) пространство $C_{p}(X)$ наследственно сепарабельно;

(c) пространство $C_{p}(X)$ наследственно секвенциально сепарабельно;

(d) пространство $X$ метризуемо.

ДокАЗАтЕЛЬСтво. Пусть $X$ метризуемо. Тогда оно компактно. По известной теореме анализа пространство $C(X)$ сепарабельно в равномерной топологии (порожденной sup-нормой); следовательно, наследственно сепарабельно, а следовательно, наследственно секвенциально сепарабельно в этой метрической топологии. Тогда $C(X)$ будет наследственно секвенциально сепарабельньм и в более слабой топологии поточечной сходимости.

Если $C_{p}(X)$ сепарабельно, то по известной теореме [3, I.1] $X$ уплотняется на пространство со счетной базой; уплотнение псевдокомпакта на пространство с первой аксиомой счетности есть гомеоморфизм, так что $X$ метризуемо.

Теорема доказана.

Из теоремы вытекает

СлЕДСТВИЕ 3. Для банахова пространства Е в слабой топологии следующие утверждения әквивалентны:

(а) пространство Е сепарабельно; 
(b) пространство Е наследственно сепарабельно;

(c) пространство Е наследственно секвенциально сепарабельно;

(d) замкнутый единичный шар $B$ сопряженного пространства $E^{\prime}$ метризуем в E-топологии.

Следствие обосновьвается на том, что

1) банахово пространство $E$ в слабой топологии представимо как замкнутое подмножество $C_{p}(B)$, где $B$ - замкнутая единичная сфера сопряженного пространства $E^{*}$

2) $B$ компактно в $E$-топологии (по теореме Алаоглу [4, V.4]);

3) справедлива теорема 2.

Следующее предположение не зависит от аксиом $Z F C$.

(В) Каждое несчетное сепарабельное метрическое пространство имеет неборелевские подмножества.

Теорема 3. (В) Если $X$ метризуемо, то $C_{p}(X)$ секвенииально сепарабельно тогда и только тогда, когда $X$ сепарабельно.

ДокАЗАТЕЛЬСТВО. В одну сторону утверждение очевидно.

Пусть $X$ несепарабельно, $F: X \rightarrow Y$ - уплотнение на пространство со счетной базой. Найдется в $X$ несчетное дискретноеподпространство $X^{\prime}$. Отображение $\left.F\right|_{X^{\prime}}$ уплотняет $X^{\prime}$ на несчетное подпространство $Y^{\prime}$ пространства $Y$. Пусть $B$ - неборелевское подмножество $Y^{\prime}, A=F^{-1}(B)$. Найдется открытое в $X$ множество $H$ такое, что $H \cap X^{\prime}=A$ (так как $A$ открыто в $X^{\prime}$ в силу дискретности $X^{\prime}$ ). Заметим, что $F(H)$ не может быть $F_{\sigma}$-множеством в $Y$, ибо в противном случае и $B$ было бы $F_{\sigma}$-множеством в $Y^{\prime}$. Доказано, что $C_{p}(X)$ не является секвенциально сепарабельным.

Вопрос. Можно ли убрать из формулировки теоремы 3 условие существования неборелевских множеств в каждом несчетном сепарабельном метрическом пространстве?

Заметим, что метризуемое пространство веса $\geqslant c$ (где $c$ - мощность континуума) не может быть секвенциально сепарабельным. Действительно, в таком пространстве имеется дискретное семейство открытых множеств мошности $\geqslant c$, а следовательно, мощность семейства всех открытых множеств $\geqslant 2^{c}$, что несовместимо - в силу теоремы 1 - с секвенциальной сепарабельностью (мощность семейства борелевских подмножеств сепарабельного метрического пространства $\leqslant c)$.

Применим теорему 1 к некоторьм конкретньм пространствам, относящимся к классике общей топологии.

Базу топологии стрелки на $\mathbb{R}$ образуют полуинтервалы $[a, b) . \mathbb{R}$ в этой топологии обозначим через $S$.

Теорема 4 (М.А. Патракеев). Пространство $C_{p}(S)$ секвенииально сепарабельно.

ДокАЗАТЕЛЬСТво. Пусть $F: S \rightarrow \mathbb{R}$ - естественное уплотнение. Любое открытое множество в $S$ есть дизъюнктное объединение счетного числа множеств, каждое из которых есть либо интервал, либо полуинтервал, которые, очевидно, являются $F_{\sigma}$-множествами в $\mathbb{R}$. Снова вьполняются условия теоремы 1.

И последнее приложение относится к пространству Немьщкого $\mathcal{N}$. 
ТЕОРема 5. Пространство $C_{p}(\mathcal{N})$ секвенциально сепарабельно.

ДокАЗАТЕЛЬСТво. Напомним, что $\mathcal{N}$ - это верхняя полуплоскость $\{(x, y): x, y \in \mathbb{R}$, $y \geqslant 0\}$. Через $\mathscr{N}_{0}$ обозначим множество $\{(x, y): y=0\}, \rho$ - естественная метрика плоскости. Базу топологии Немьщкого на $\mathscr{N}$ образуют множества $B(x, \varepsilon) \cap \mathscr{N}$ такие, что $B(x, \varepsilon)$ - открытьй шар радиуса $\varepsilon$ с центром в точке $x$ при $x \notin \mathscr{N}_{0}$, и $B(x, \varepsilon)=$ $B_{0}(x, \varepsilon) \cup\{x\}$ при $x \in \mathscr{N}_{0}$, где $B_{0}(x, \varepsilon)$ - открытый шар радиуса $\varepsilon$, касаюшийся оси $\mathscr{N}_{0}$ в точке $x$.

Пусть $\tau$ - естественная топология полуплоскости $\mathscr{N}, U-$ конуль-множество $\mathscr{N}$. Если множество $U \subseteq \mathscr{N} \backslash \mathscr{N}_{0}$, то оно $\sigma$-компактно в $(\mathscr{N}, \tau)$, тем более, является $F_{\sigma}$-множеством в $(\mathscr{N}, \tau)$. Тоже самое можно сказать об $U$, если множество $U \cap \mathscr{N}_{0}$ счетно. Остается рассмотреть случай, когда $U \cap \mathscr{N}_{0}$ несчетно.

Найдется непрерывное отображение $f: \mathscr{N} \rightarrow[0,1]$ такое, что $U=f^{-1}((0,1])$. Положим $F_{n}^{\prime}=(1 / n, 1]$ и $F_{n}=f^{-1}\left(F_{n}^{\prime}\right) \cap \mathscr{N}_{0}$. Тогда $U \cap \mathscr{N}_{0}=\bigcup\left\{F_{n}\right\}$. Фиксируем $n$. Если $F_{n}$ счетно, то положим $S_{n}=F_{n}$. Пусть $F_{n}$ несчетно. Для каждой точки $x \in F_{n}$ находим число $k=k(x)$ такое, что $B(x, 1 / k) \subseteq f^{-1}\left(F_{n}^{\prime}\right)$. Положим $r(x)=k$ и $W_{n, k}=\{x: r(x)=k\}$. Если $W_{n, k}$ счетно, то положим $S_{n, k}=W_{n, k}$. Пусть $W_{n, k}$ несчетно. Тогда найдется счетное множество $S_{n, k}^{\prime} \subseteq W_{n, k}$ такое, что $W_{n, k} \backslash S_{n, k}^{\prime}$ плотно в себе (в топологии $\tau$, по теореме Кантора-Бендиксона). Тогда $\left[W_{n, k} \backslash S_{n, k}^{\prime}\right]_{\tau} \subseteq U$.

Действительно, пусть $x \in\left[W_{n, k} \backslash S_{n, k}^{\prime}\right], B(x, \varepsilon)$-произвольная базисная окрестность точки $x$ в пространстве $\mathcal{N}$. Найдется $y \in W_{n, k} \backslash S_{n, k}^{\prime}$ такое, что либо $\rho((y, 2 / k),(x, \varepsilon))<\varepsilon$ (при $2 / k<\varepsilon$ ), либо $\rho(y, x)<\varepsilon$ (при $\varepsilon<2 / k)$. Очевидно, что $B(x, \varepsilon) \cap B(x, 1 / k) \neq \varnothing$. Из этого следует, что $f(x)>0$ и $x \in U$. Положим $S_{n, k}=S_{n, k}^{\prime} \cup\left[W_{n, k} \backslash S_{n, k}^{\prime}\right]_{\tau} ;$ получим $F_{\sigma}$-множество в $\tau$. Тогда $F_{\sigma}$-множеством будет и $S_{n}=\bigcup\left\{S_{n, k}: k \in \mathbb{N}\right\}$. А так как $U \cap \mathscr{N}_{0}=\cup\left\{F_{n}: n \in \mathbb{N}\right\}, F_{n}=\bigcup\left\{W_{n, k}: k\right\}$ и $W_{n, k} \subseteq S_{n, k} \subseteq U$, то $F_{\sigma}$-множествами будут и $U \cap \mathscr{N}_{0}=\cup\left\{S_{n}: n \in \mathbb{N}\right\}$, и само множество $U$.

Остается применить теорему 1.

Теорема 5 доказана.

Заметим, что пространства $C_{p}(S)$ и $\mathscr{N}$ не являются наследственно сепарабельными (в силу теоремы автора [3, II.5]), так что мы имеем примеры пространств $C_{p}(X)$, которые секвенциально сепарабельны, но не являются наследственно (секвенциально) сепарабельньми.

\section{СПИСОК ЦИТИРОВАННОЙ ЛИТЕРАТУРЫ}

[1] Gartside P., Lo J. T.H., Marsh A. Sequential density // Topology Appl. 2003. V. 130. P. $75-86$.

[2] Куратовский К. Топология. Т. 1. М.: Мир, 1966.

[3] Архангельский А. В. Топологические пространства функций. М.: Изд-во МГУ, 1989.

[4] Данфорд Н., Шварц Д. Т. Линейные операторы. Общая теория. М.: ИЛ, 1962.

Институт математики и механики УрО РАН

E-mail: vel@imm.uran.ru

Поступило 18.05 .2004 Исправленный вариант 05.04 .2005 